Supporting Information

\title{
Multilayer Carbon Nanotube/Gold Nanoparticle Composites on Gallium-Based Liquid Metals for Electrochemical Biosensing
}

Taehwan Lim ${ }^{1}$, Huanan Zhang ${ }^{1, *}$

1 Department of Chemical Engineering, the University of Utah, Salt Lake City, UT

*Author to whom correspondence should be addressed: huanan.zhang@utah.edu 


\section{CNT/PDDA deposition}

by negative potential
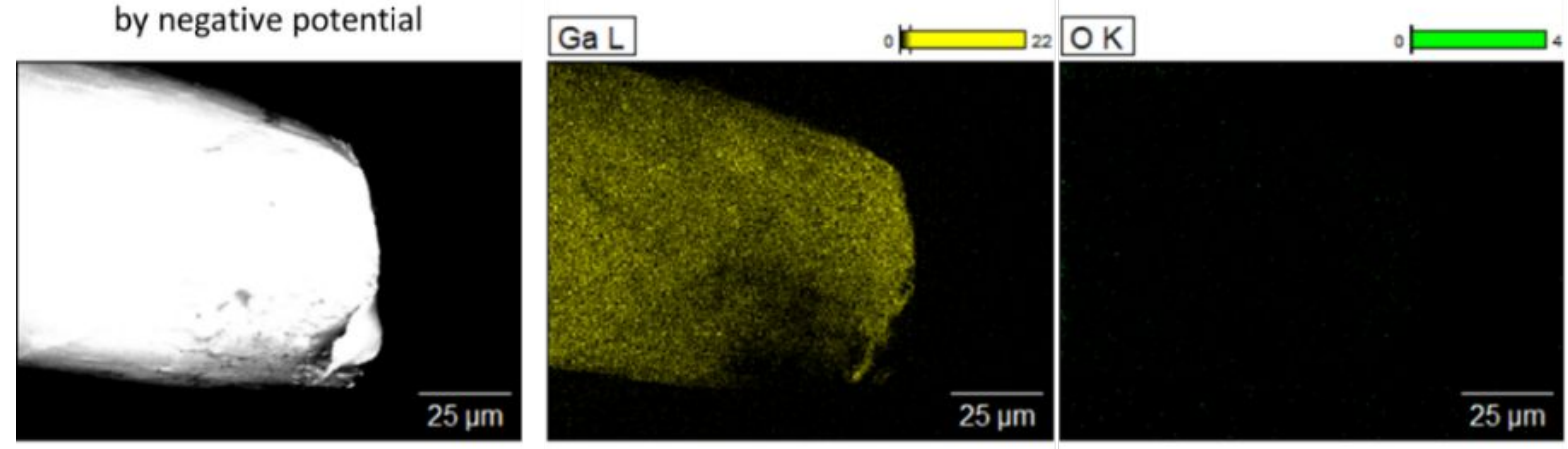

*PEBAX wall dissolved in THF for $5 \mathrm{~min}$

Figure S1. SEM image by backscattered electrons (left) and EDS analysis to confirm oxide layer formation after thermal stretching process (middle: Gallium, right: Oxygen). 

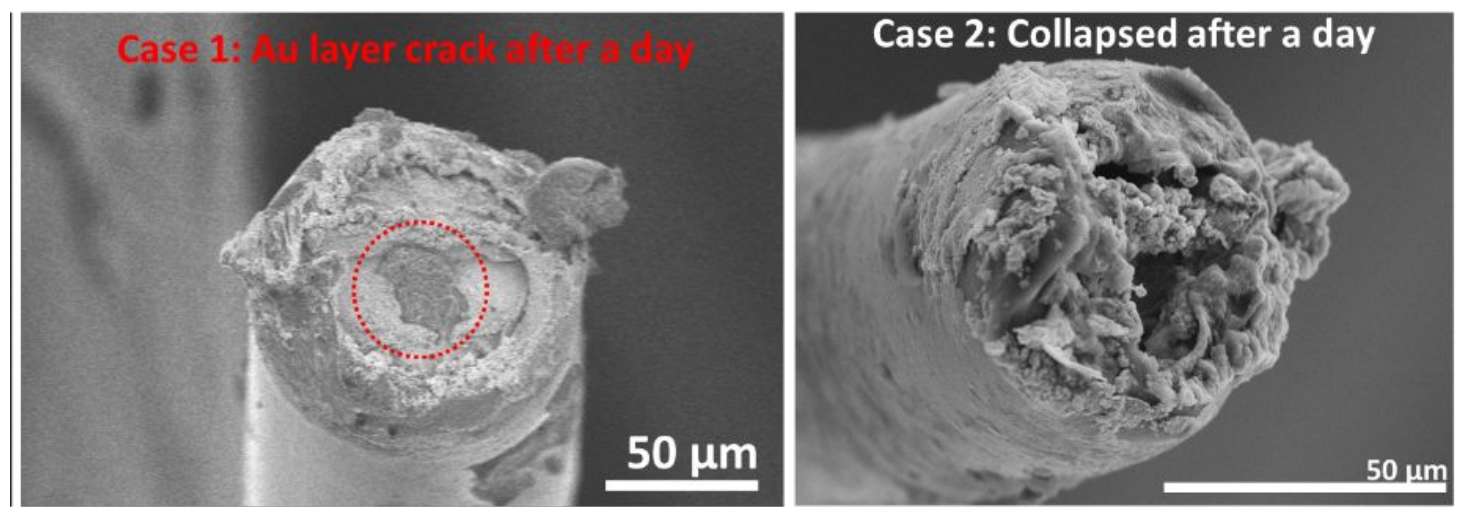

Figure S2. SEM images of cracked (left) and collapsed (right) Au NPs surface on the Ga based liquid metal electrode when kept in PBS a day or bended a wire after liquefying the electrode. 

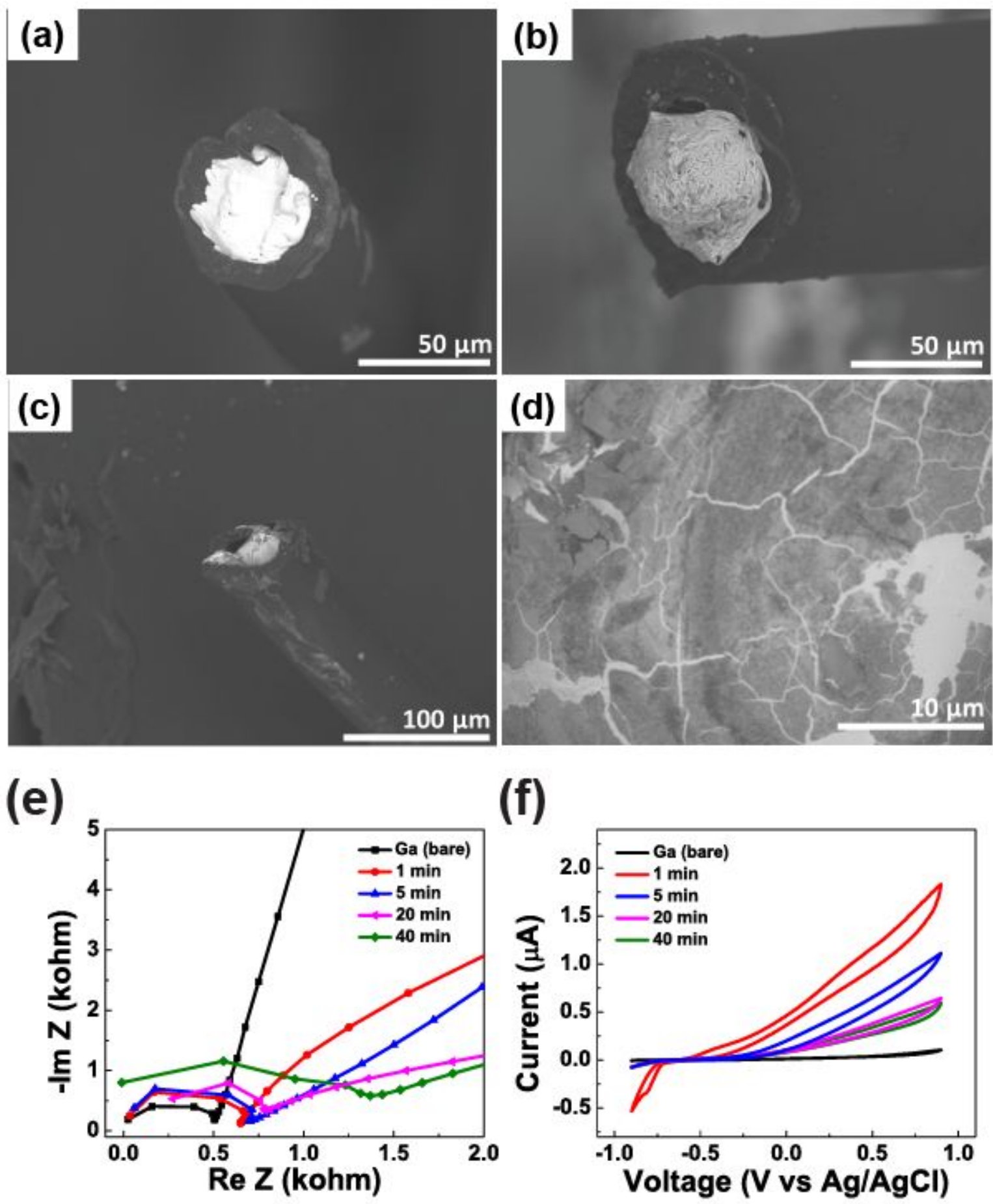

Figure S3. SEM images of (a) bare, (b) $20 \mathrm{~min}$, and (c) 40 min oxidized Ga wire with $+2.5 \mathrm{~V}$ in PBS at $37^{\circ} \mathrm{C}$. (d) x10 magnified c. All the images were obtained from BSE mode. (e) Nyquist impedance and (f) $\mathrm{CV}$ curves of oxidized $\mathrm{Ga}$ wires with electrochemical oxidation until $40 \mathrm{~min}$ $\left(+2.5 \mathrm{~V}\right.$ chronoamperometry) at $37^{\circ} \mathrm{C}$. 

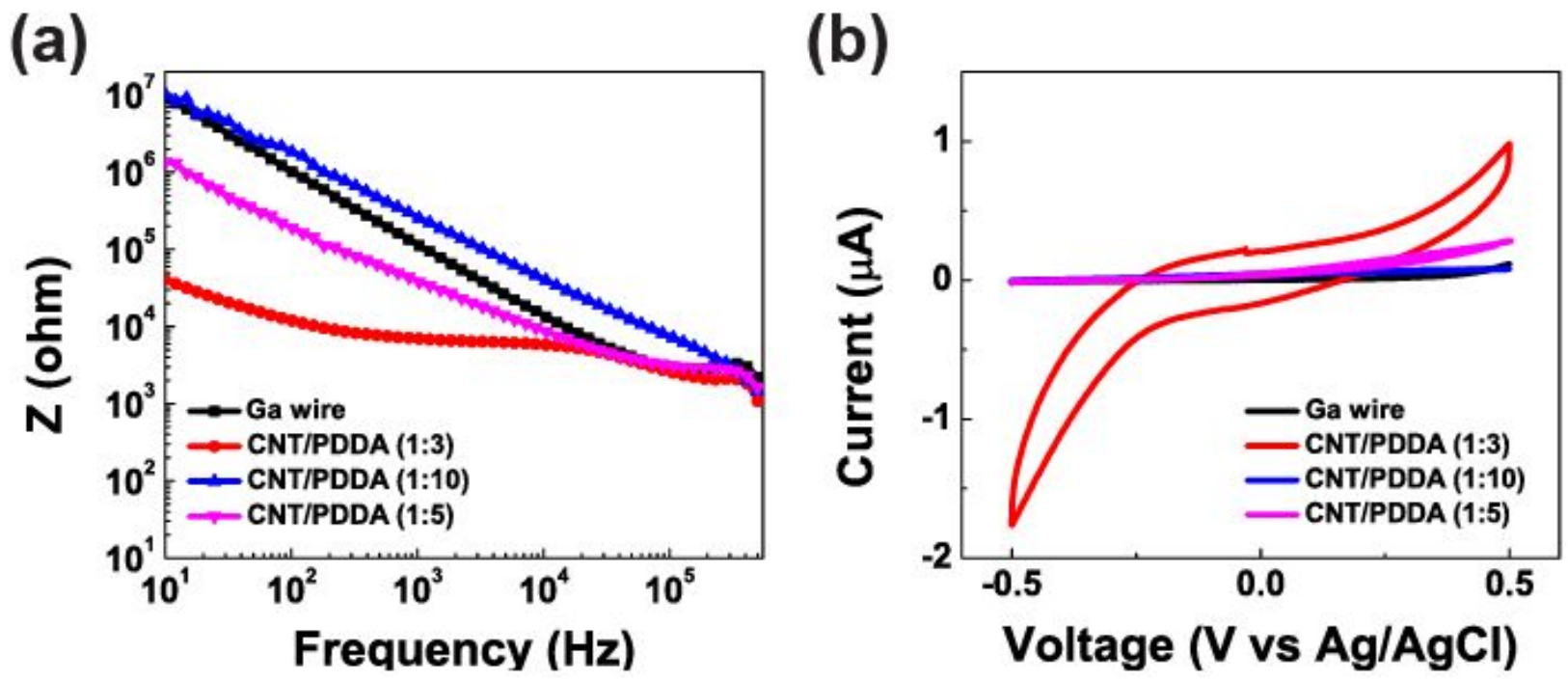

Figure S4. (a) Bode impedance and (b) CV curves of microelectrodes after CNT/PDDA deposition on $\mathrm{Ga}$ with different ratios between CNT and PDDA for $40 \mathrm{~min}$. 

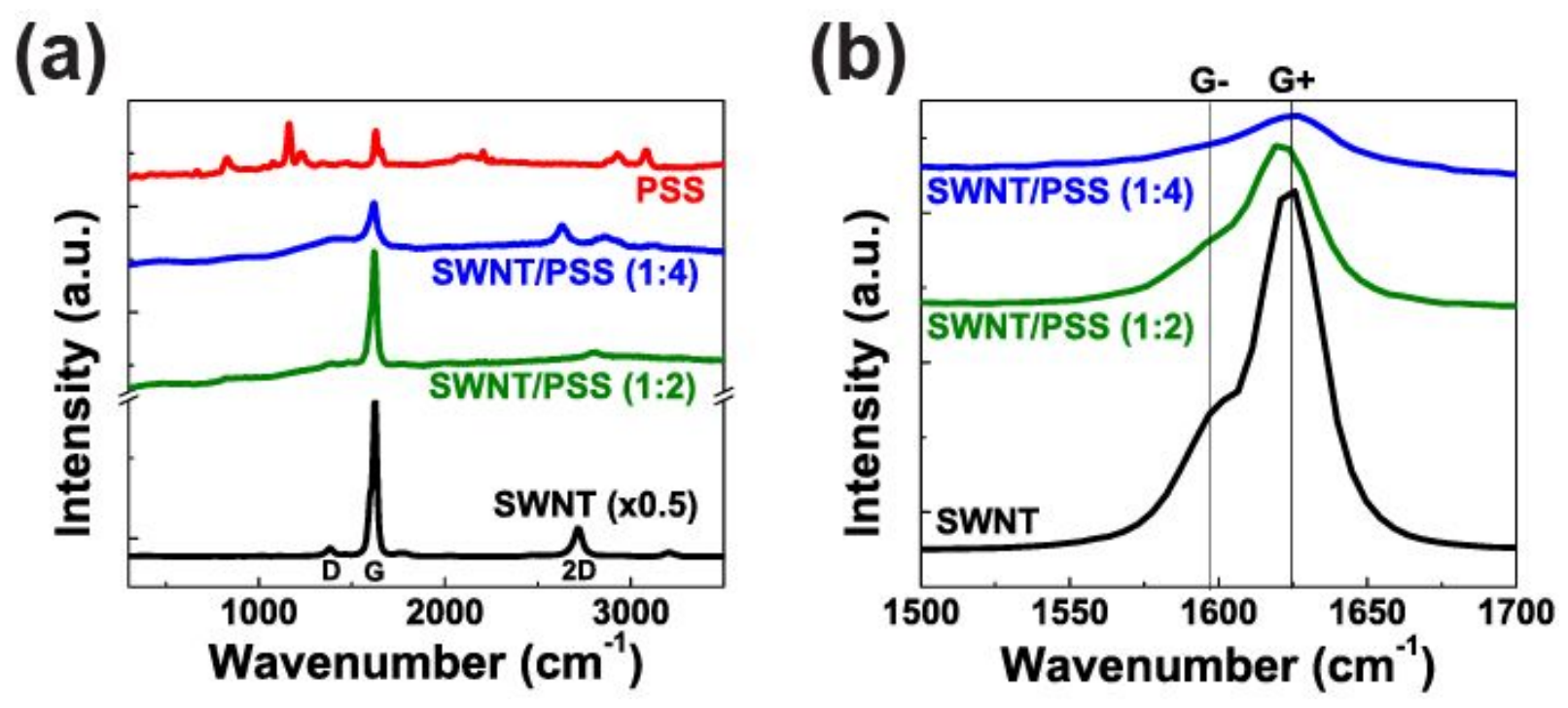

Figure S5. (a) RAMAN spectra of SWNT and SWNT/PSS composites with ratios. (b) G band of RAMAN spectra of SWNT/PSS with the ratios. 

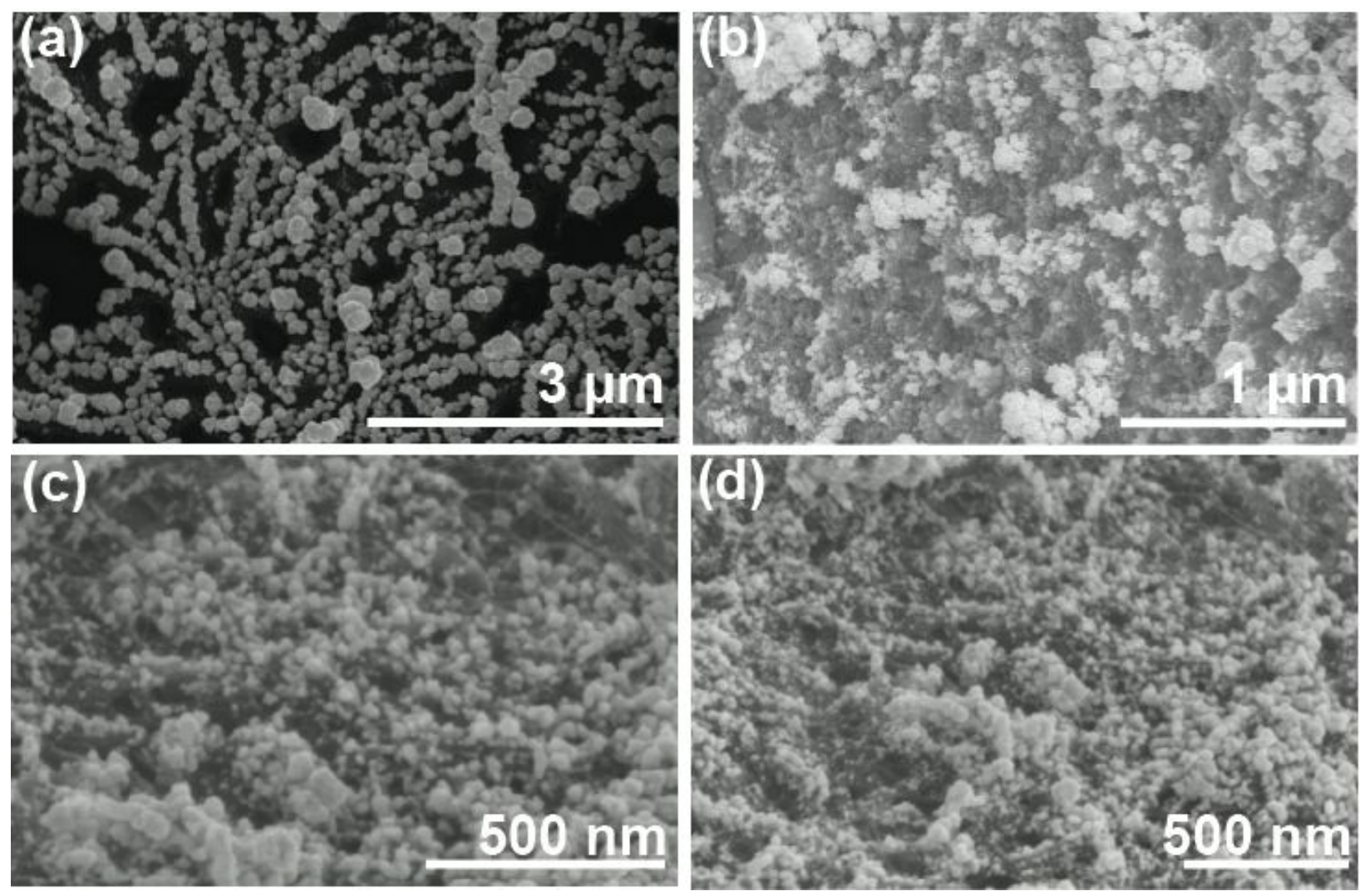

Figure S6. SEM images of (a) Au decorated CNT and Au NPs on the CNT/PDDA layer with the electrochemical deposition of (b) $3 \mathrm{~min}$, (c) $5 \mathrm{~min}$, and (d) $10 \mathrm{~min}$. 

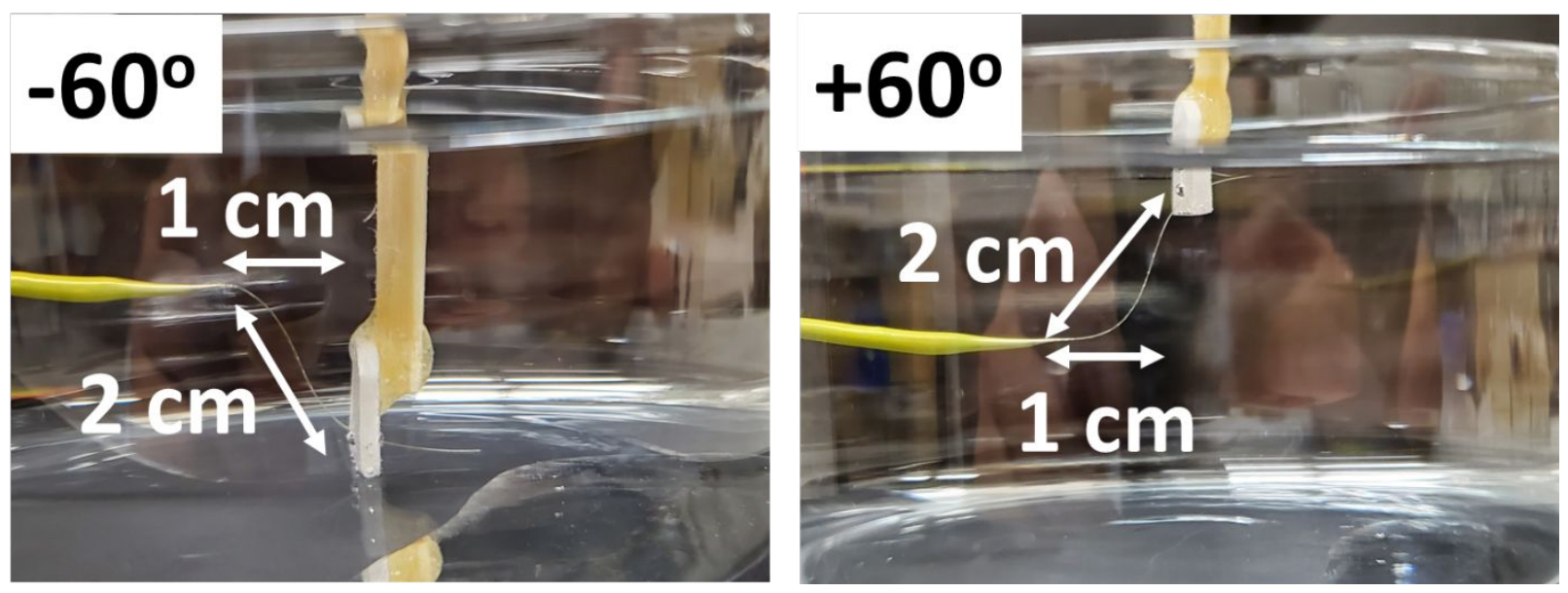

Figure S7. SEM images of (a) Au decorated CNT and Au NPs on the CNT/PDDA layer with the electrochemical deposition of (b) $3 \mathrm{~min}$, (c) $5 \mathrm{~min}$, and (d 

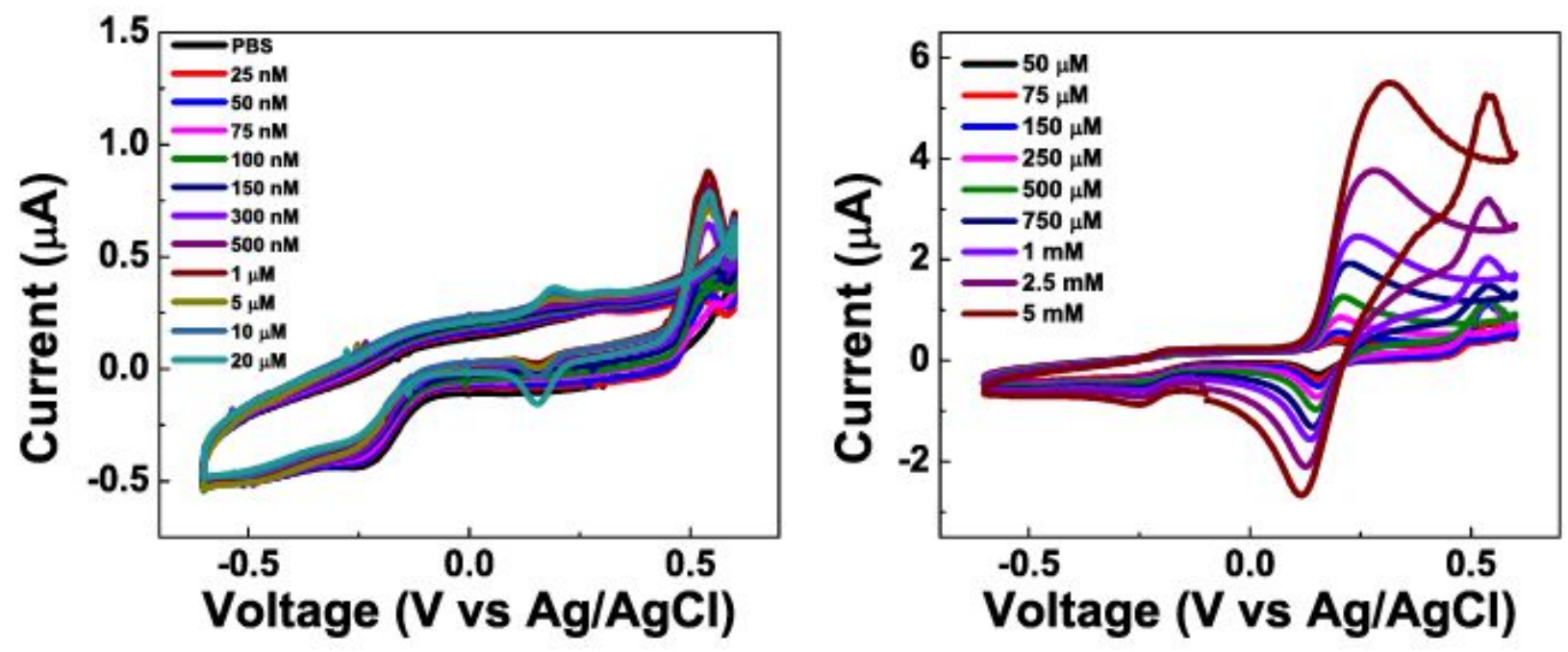

Figure S8. CV curves with the amounts of DA concentrations for sensitivity confirmation. 
(a)

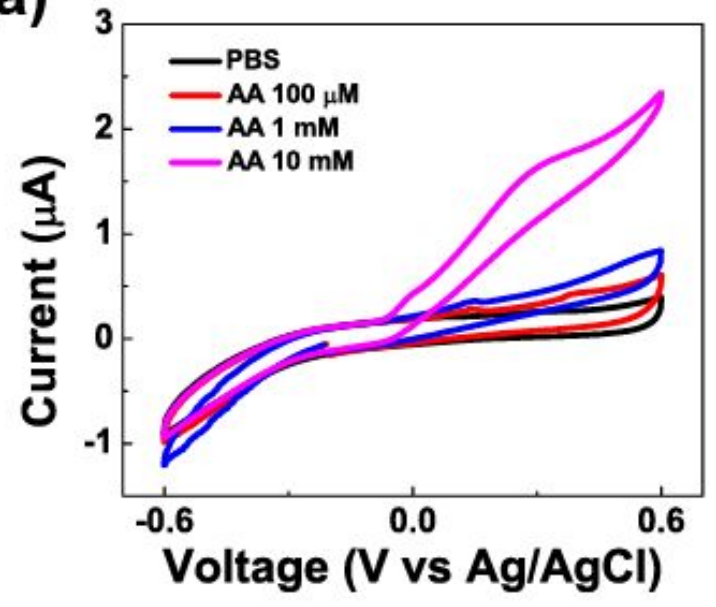

(b)

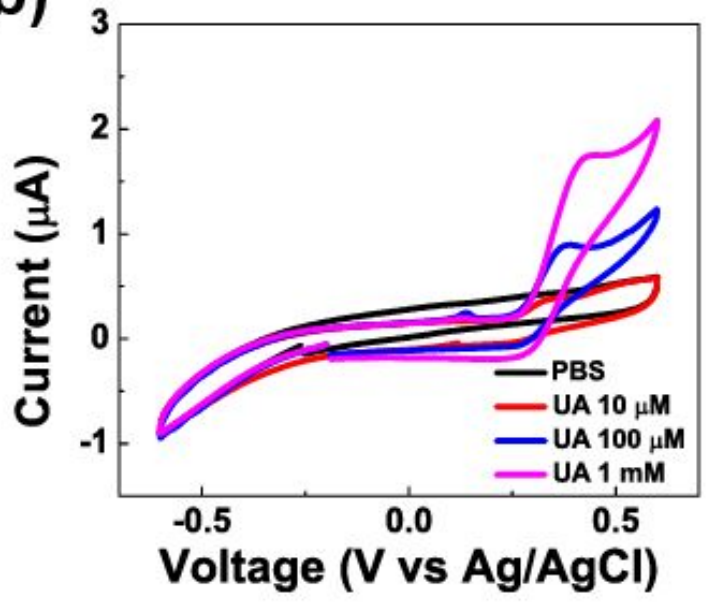

Figure S9. CV curves with the amounts of (a) AA and (b) UA. 

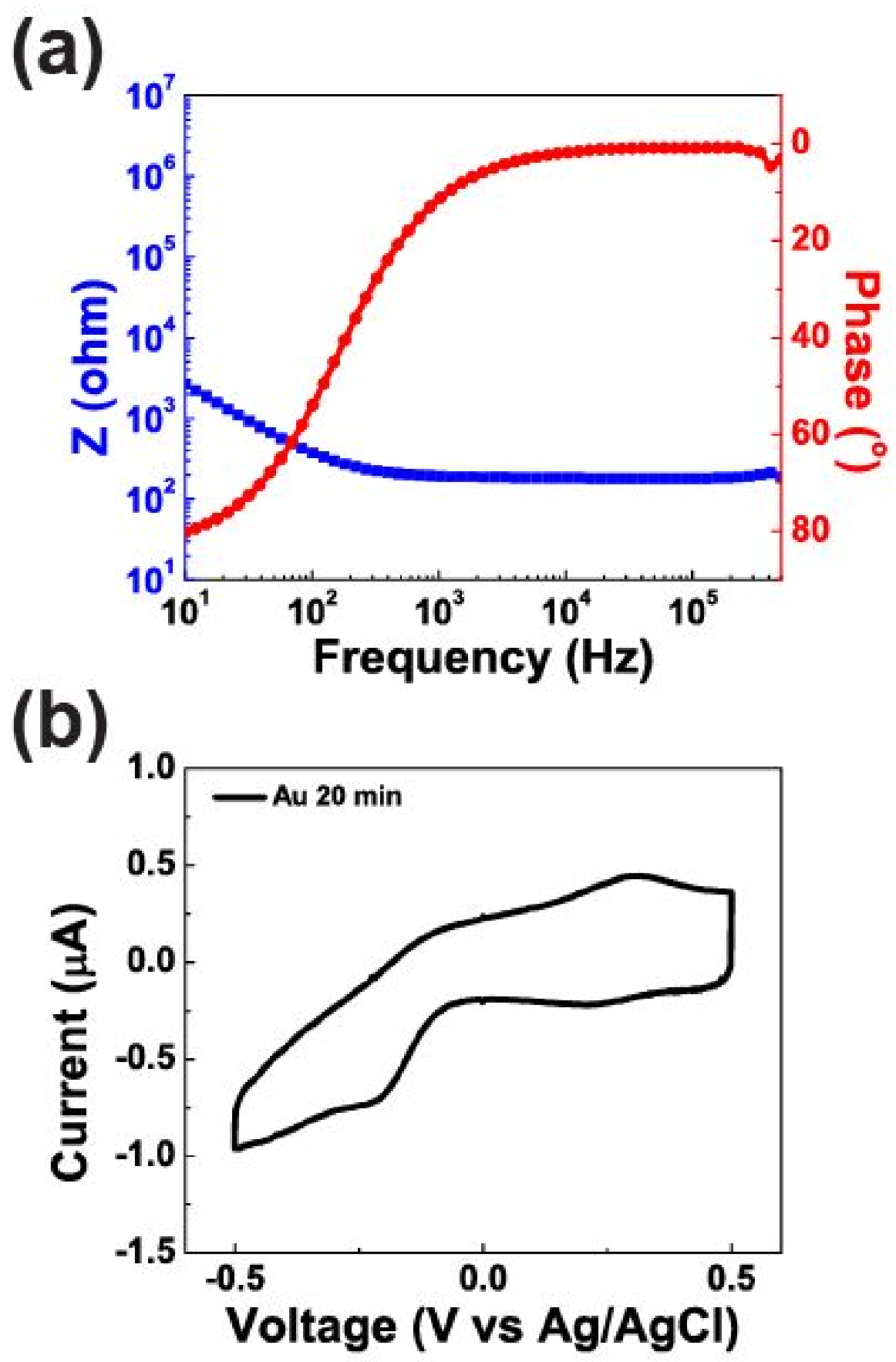

Figure S10. Electrochemical properties of nanostructure functionalized liquid metal electrodes in ACSF; (a) bode impedance and (b) CV curves with CNT and Au deposition time. 

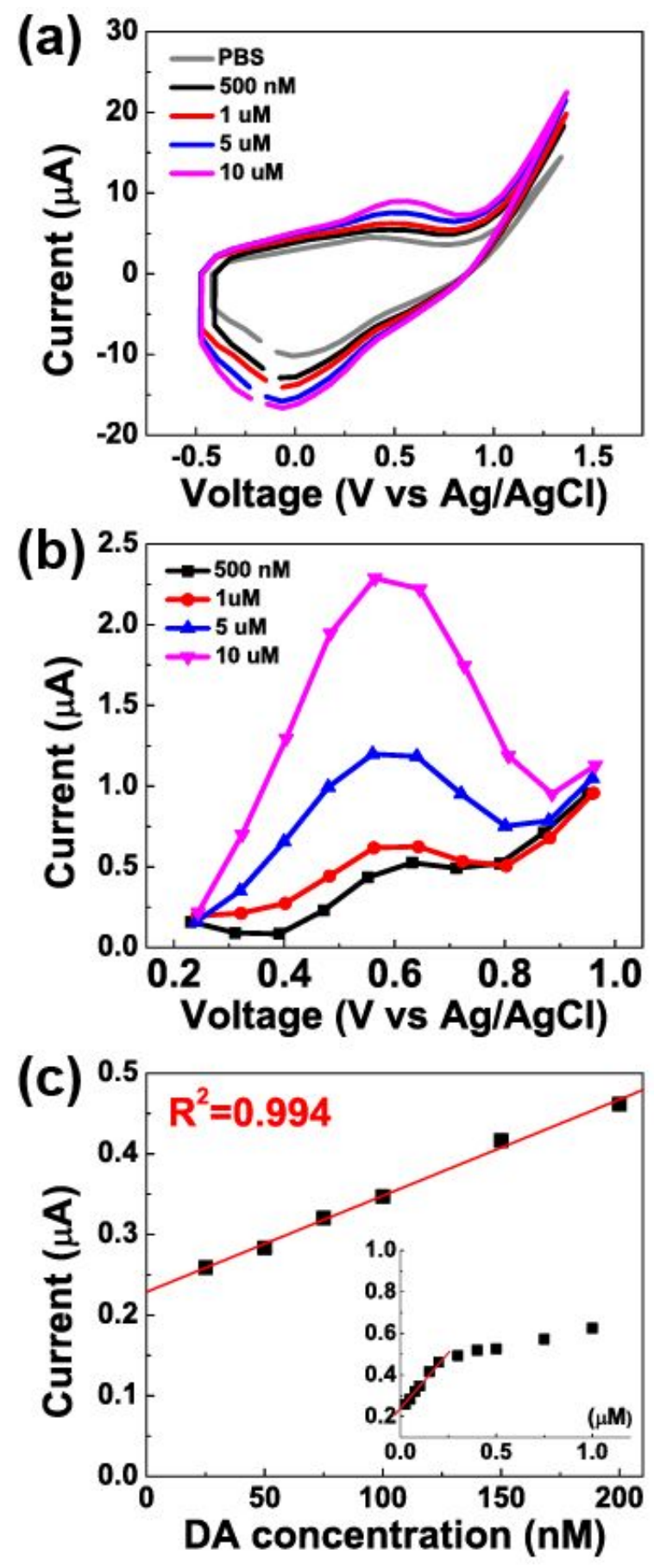

Figure S11. FSCV test results with the amounts of DA in 0.1M PBS; (a) with background and (b) after background subtraction. (c) a linear fit of anodic peaks with the amounts DA under $200 \mathrm{nM}$ range to determine the sensitivity from FSCV test. 